\title{
Análise de Soft Skills na Visão de Profissionais da Engenharia de Software
}

\author{
Tiago de Lima ${ }^{1}$, Josiane Brietzke Porto ${ }^{1}$ \\ ${ }^{1}$ Educação Online - Universidade do Vale dos Sinos (UNISINOS) \\ Av. Unisinos, 950 - 93.022-750 - São Leopoldo - RS - Brasil \\ lima.tiago@hotmail.com, josibrietzke@unisinos.br
}

\begin{abstract}
A career in Software Engineering demands the development of several technical skills. However, these professionals also need to develop non-technical skills related to soft skills. Through an electronic survey, this research raised the importance of soft skills and which have the greatest impact on career development and daily activities, based on the perception of professionals in this area. The results showed that these professionals understand that soft skills have a high impact on the development of their careers and daily activities. However, there is no greater concern with their development when considering the sample of this research.
\end{abstract}

Resumo. A carreira na Engenharia de Software demanda desenvolvimento de diversas competências técnicas. Entretanto, esses profissionais também precisam desenvolver competências não técnicas, relacionadas às soft skills. Por meio de survey eletrônico, essa pesquisa levantou a importância de soft skills e quais possuem maior impacto no desenvolvimento de carreira e em atividades diárias, a partir da percepção de profissionais dessa área. Os resultados mostraram que esses profissionais entendem que as soft skills possuem alto impacto no desenvolvimento de suas carreiras e em suas atividades diárias. Porém, não existe uma preocupação maior com o desenvolvimento delas, quando se considera a amostra dessa pesquisa.

\section{Introdução}

O desenvolvimento de uma carreira profissional está relacionado às competências desenvolvidas pelo indivíduo, com a finalidade de que este esteja preparado para lidar com os diferentes desafios de uma organização. Os processos de globalização, a turbulência crescente e a complexidade maior das arquiteturas organizacionais criaram a necessidade de pessoas mais autônomas e com maior iniciativa [Dutra, 2010]. O conceito de competência foi proposto de forma estruturada pela primeira vez em 1973, por David McClelland (1973), na busca de uma abordagem mais efetiva que os testes de inteligência, nos processos de escolha de pessoas para as organizações [Dutra, 2010].

A Engenharia de Software é uma disciplina da engenharia cujo foco está em todos os aspectos da produção de software, desde os estágios iniciais da especificação do sistema até sua manutenção, quando o sistema já está sendo usado. Visa obter resultados de qualidade requeridos dentro do cronograma e do orçamento [Sommerville, 2011]. Em geral, engenheiros de software adotam uma abordagem sistemática e organizada para seu trabalho, pois essa costuma ser a maneira mais eficiente de produzir 
software de alta qualidade. No entanto, engenharia tem tudo a ver com selecionar o método mais adequado para um conjunto de circunstâncias, então, uma abordagem não criativa e menos formal, pode ser eficiente em determinadas circunstâncias. O engenheiro de software necessita desenvolver uma série de competências técnicas relacionadas com o desenvolvimento de software, gerenciamento de projetos, teste de software, entre outras para o desenvolvimento de suas atividades diárias.

Peters e Pedrycz (2001) citam que a cada ano são frequentes novas versões de produtos de software existentes, de novos produtos e de tecnologias de software. Isto demanda constante atualização por parte do engenheiro de software. Alertam que uma compreensão dos fatores humanos no processo de desenvolvimento de software é crucial para a organização de equipes dessa área e para o estímulo, no sentido de assumir uma abordagem disciplinada ao desenvolvimento de software.

Sommerville (2011) defende que a maior parte dos softwares profissionais é desenvolvida por equipes e que uma série de fatores influenciam o trabalho em grupo: (i) composição do grupo: precisa de um equilíbrio de habilidades, experiências e personalidades; (ii) coesão do grupo: se este pensa em si como uma só equipe e não como um conjunto de indivíduos trabalhando juntos; (iii) comunicação dentro do grupo: se os membros se comunicam eficazmente uns com os outros; (iv) organização do grupo: organizado de forma que todos se sintam valorizados e satisfeitos. Desta forma, não somente competências técnicas são importantes para que o engenheiro de software tenha uma carreira de sucesso. Existe a necessidade do desenvolvimento de competências não técnicas, como às relacionadas ao trabalho em grupo, comunicação, resiliência, entre outras, denominadas soft skills.

O desenvolvimento de sistemas de software complexos requer uma mistura de competências técnicas e não técnicas e, enquanto existem algumas diretrizes referentes ao conhecimento técnico necessário para se tornar um bom engenheiro de software, existe uma carência de conhecimento sobre quais competências não técnicas são necessárias para dominar projetos complexos de software [Sedelmaier e Landes, 2014]. Nesse sentido, Ahmed et al. (2013) analisaram que existe demanda por determinadas competências do ponto de vista da indústria de software, como capacidade de comunicação, habilidades interpessoais, capacidade analítica e de resolução de problemas, trabalho em equipe, organização, habilidade para trabalhar de forma independente, habilidade de ser aberto e adaptável a mudanças.

Diante da importância de soft skills na Engenharia de Software, esse estudo investigou a questão: Na visão de profissionais da Engenharia de Software, quais as soft skills mais importantes e que possuem maior impacto no desenvolvimento de suas carreiras e atividades diárias? Para tanto, essa pesquisa identificou e analisou essas competências não técnicas consideradas como importantes, tendo como base a percepção de profissionais atuantes nessa área.

Torna-se relevante por apresentar a visão dos próprios profissionais atuantes na área, uma visão complementar e de certa forma diferente de trabalhos anteriores revisados (item 2.2), que mostraram uma visão acadêmica e da indústria de software sobre a necessidade do desenvolvimento de competências não técnicas, apresentando as mais requisitadas pelo mercado de trabalho. Contribui para organizações intensivas em desenvolvimento de software, pois seus achados podem ajudar na concepção de 
iniciativas de qualificação e de capacitação de colaboradores para desenvolver tais competências, buscando minimizar a influência das soft skills sobre atividades associadas à Engenharia Software. A partir do conhecimento de tais competências, as organizações podem trabalhar para criar uma cultura de desenvolvimento pessoal, obtendo como benefícios o sentimento de realização profissional e, por consequência, conseguir retenção de seus talentos.

Esse estudo é importante para que os profissionais planejem e estejam mais qualificados para o mercado de desenvolvimento software, não somente pelas suas competências técnicas, mas igualmente pelas competências não técnicas identificadas. Esse levantamento da visão dos profissionais é relevante, pois seus resultados podem contribuir para reflexão pelos responsáveis em instituições de ensino e construção de projetos de cursos direcionados às soft skills, nos diversos níveis de formação.

Além da introdução, esse artigo é organizado em mais 4 seções. Na seção 2 são abordados conceitos de Engenharia de Software e competências não técnicas. Na seção 3, os aspectos metodológicos adotados nessa pesquisa são apresentados. Na seção 4, os resultados são apresentados e analisados. Por fim, a seção 5 traz considerações finais.

\section{Soft Skills na Engenharia de Software}

\subsection{Engenharia de Software}

A Engenharia de Software é uma disciplina de importância crítica para o futuro da humanidade e os engenheiros de software devem continuar a serem educados para desenvolver competências referentes a essa área de conhecimento, para que possam criar sistemas de software cada vez mais complexos [Sommerville, 2011]. Entretanto, trata-se de uma disciplina em amadurecimento, que estuda metodologias e abordagens para o desenvolvimento de software em larga escala.

No início, a Engenharia de Software era voltada para uma profissão orientada ao laboratório e, por volta do ano 2000, passou para ser mais orientado a indústria [Wang e King, 2000]. Historicamente focou em metodologias e linguagens de programação, modelos e ferramentas de desenvolvimento de software. Concentrava-se em aspectos puramente técnicos e áreas consideradas críticas para a Engenharia de Software eram amplamente ignoradas, como as infraestruturas organizacionais e de gerenciamento, que apoiam essa produção de software, atualmente.

Numa abordagem de engenharia, a Engenharia de Software caracteriza-se por um desenvolvimento de software prático, ordenado e medido, que respeita prazos, orçamentos e qualidade. As pessoas que trabalham em uma organização de software são seu maior patrimônio. Representam o capital intelectual e é responsabilidade dos gerentes de software garantir obtenção do melhor retorno de investimentos em pessoas. Em empresas e economias bem-sucedidas, isso é alcançado quando as pessoas são respeitadas pela organização [Sommerville, 2011].

Nessa área, quando gerentes tomam decisões acerca dos envolvidos em determinado projeto de longo prazo devem levar em conta habilidades de solucionar problemas em geral e a experiência de domínio, além das habilidades técnicas e específicas de linguagem de programação e arquitetura da solução [Sommerville, 2011]. 


\subsection{Soft Skills do Engenheiro de Software}

Competência pode ser entendida como conjunto de conhecimentos, habilidades e atitudes. Pode ser percebida como um estoque de recursos, que o indivíduo detém. A competência do indivíduo não é um estado, não se reduz a um conhecimento ou knowhow específico [Fleury, 2001].

Calazans et al. (2017) compararam visões entre academia e mercado de trabalho no que tange às competências do engenheiro de requisitos. Gimenes (2015) destacou dilemas envolvidos nas disciplinas de Engenharia de Software e entre estes está o fato de os professores serem conteudistas, o que implica que não são trabalhadas soft skills como comunicação, liderança, resolução de conflitos e dinâmica de grupo, sendo estas habilidades imprescindíveis para engenheiros de software, segundo a autora.

Sedelmaier e Landes (2014) apresentaram grupos de competências necessárias para um engenheiro de software, como a colaboração. Somente aqueles indivíduos que são capazes de colaborar com outros seres humanos são capazes de resolver os problemas que são colocados aos engenheiros de software, diariamente. A comunicação é um meio necessário e inevitável de troca de informações sempre que os humanos colaboram. Os autores chamam de estrutura, a necessidade de engenheiros de software se estruturarem e trabalharem num ambiente complexo e de competências pessoais, aquelas que visam autorreflexão e manejo consciente e objetivo de desafios e obstáculos individuais.

Para Sedelmaier e Landes (2014), capacidade de compreender processos, sistemas e relacionamentos complexos refere-se a abstrair configurações complexas de problemas e concretizar possíveis soluções. Os engenheiros de software precisam estar dispostos e capazes de pensar fora da caixa e de entender e aceitar a significação e a necessidade de procedimentos e artefatos supostamente estranhos. Competência para resolver problemas e situações novas. E, ainda, competências adicionais, que englobam responsabilidade pelos outros e por projetos conjuntos, pesquisas, adaptações e uso de informações identificadas para resolver problemas, comportamento conforme normas sociais e éticas e, por fim, capacidades de se expressar por escrito adequadamente.

Diferentes estudos apresentaram classificações distintas para soft skills. Rao (2012) entende que são competências intrapessoais e interpessoais, que determinam a capacidade de uma pessoa em ter um bom relacionamento com os outros e se destacar no mundo corporativo. González-Morales et al. (2011) encontrou as seguintes soft skills: trabalho em equipe; liderança; responsabilidade; aprendizagem autônoma; moral ética e profissional; gerenciamento de projetos; planejamento; negociação; comunicação oral; habilidades interpessoais com colegas e clientes; comunicação escrita; criatividade; capacidade de aplicar conhecimento no espaço de trabalho; capacidade de aprender novas habilidades; pensamento crítico e resolução de problemas; aprendizagem ao longo da vida e gestão da informação; empreendedorismo.

Ahmed et al. (2013) mostrou que a indústria de software presta atenção às habilidades sociais enquanto contrata e que há necessidade de reconhecer mais o papel dessas habilidades no desenvolvimento de software. Concluíram que esse desenvolvimento requer uma variedade de habilidades flexíveis e difíceis para resolver 
os diversos problemas que enfrenta, bem como que a indústria de software deve entender esse relacionamento complexo.

Froehlich (2016) analisou presença e importância de soft skills no gerenciamento de projetos de service desk, a partir da percepção de profissionais da área. Pode concluir que de uma maneira geral há um maior nível de percepção de importância das soft skills do que de presença no perfil da gestão. Os resultados justificaram projetos mal estruturados, comunicação inadequada entre os membros e a gestão do projeto, gestores despreparados e equipes desmotivadas no ciclo de vida do projeto.

A partir desses estudos anteriores se percebe que desenvolver soft skills não deve ser apenas vista pelo mercado, mas pelos responsáveis no processo de desenvolvimento acadêmico dos engenheiros de software. Caso não estejam fazendo ainda, universidades devem passar a incorporar nos currículos dos cursos, disciplinas como inovação, empreendedorismo, psicologia e gestão de pessoas, negociação e resolução de conflitos. No presente estudo aborda-se a visão de profissionais de Engenharia de Software em relação à importância das soft skills, no desenvolvimento de suas próprias carreiras. $\mathrm{Na}$ literatura revisada, esse ponto de vista ainda carece de atenção e de mais pesquisas.

\section{Método de Pesquisa}

Essa pesquisa se caracteriza como quantitativa de caráter exploratório. Para Gerhardt e Silveira (2009), a pesquisa quantitativa se centra na objetividade, considera que a realidade só pode ser compreendida com base na análise de dados brutos, recolhidos com o auxílio de instrumentos padronizados e neutros.

Para identificar soft skills na visão de profissionais da área de Engenharia de Software foi realizada uma survey eletrônica, com profissionais residentes no Brasil, que estavam exercendo atividades relacionadas à Engenharia de Software há um ano ao menos, numa amostra não probabilística, obtida por conveniência e formada por 37 respostas válidas, conforme critérios de pesquisa.

Um pré-teste da versão inicial do instrumento baseado na literatura revisada (maior número de aparições nos trabalhos do item 2.2) foi feito com objetivo de antecipar e descobrir erros, revisar a estrutura e conteúdo para fins de refinamentos e geração de uma versão final [Cooper e Schindler, 2003]. Esse pré-teste ocorreu em duas etapas: (i) Pré-teste de especialista; (ii) Pré-teste de respondente.

$\mathrm{Na}$ etapa (i), dois especialistas participaram de forma remota e individual, de 8 a 13 de julho de 2018, fornecendo feedbacks, que oportunizaram ajustes na nomenclatura de duas soft skills existentes no instrumento, inclusão das soft skills "Visão do negócio", "Foco no cliente", "Empatia" e "Tomada de decisão", além de nova questão sobre frequência de uso dessas nas atividades diárias. A etapa (ii) foi de 15 a 20 de julho de 2018, com três profissionais que atuam em suporte e desenvolvimento de software. O convite para participação voluntária foi enviado para e-mail pessoal e além do preenchimento, se pediu feedback com críticas, sugestões, erros e considerações. A partir dos retornos houve alteração do tempo médio de resposta e adicionada escala para duas perguntas do instrumento.

$\mathrm{Na}$ versão final do instrumento, as primeiras questões visavam identificar as soft skills de maior importância no desenvolvimento de carreira e nas tarefas diárias em 
Engenharia Software. A segunda parte questões com objetivo de levantar o perfil dos respondentes. A divulgação ocorreu entre os dias 23 de julho e 06 de agosto de 2018, por e-mail, no perfil dos pesquisadores e em grupos da área, no Facebook (Sistemas de Informação - TI, Eng. de Software - Unisinos, Eng. de Software UTFPR-DV e Unisinos - SI, entre outros) e no LinkedIn (GUMA, Eng. de Software - IC/Unicamp, TI Brasil).

A análise dos dados foi feita primeiramente com todos os respondentes, sem considerar cargo ou experiência na Engenharia de Software. Depois, foram separados e analisados somente aqueles com mais de 1 ano de experiência. Usou-se distribuição de frequência, além de representações gráficas para análise e comparação dos dados.

\section{Análise dos Resultados}

\subsection{Perfil dos Respondentes}

Dentre do total de 41 respondentes da survey, 37 possuem mais de 1 ano de experiência na área, estando desta forma dentro da amostra definida para essa pesquisa. A maioria dos respondentes é do sexo masculino (31), está na faixa etária entre 25 e 34 anos (14), 37 trabalham em uma empresa privada ou mista (público/privada), 28 são residentes do estado do Rio Grande do Sul, sendo a maior parte analistas de suporte (16) ou desenvolvedores (10).

É possível verificar que do total de respondentes da amostra, a maioria possui uma formação acadêmica relacionada à área de Tecnologia da Informação (34), mas existem algumas exceções com formações em Administração de Empresas e Engenharia Elétrica, por exemplo. Do total, a maioria possui nível de formação em Pós-graduação Lato Sensu (MBA/Especialização) (16).

\subsection{Análise da Importância no Desenvolvimento de Carreira}

$\mathrm{Na}$ Questão 1, o objetivo foi medir o nível de importância das soft skills para o desenvolvimento de carreira (Likert de 5 opções e SCO - Sem condições de opinar). Essas competências foram percebidas de forma geral entre importante, muito importante e essencial, com destaque para "Trabalhar em equipe", "Responsabilidade", e "Moral ética e profissional", conforme Tabela 1.

Esses resultados convergem com a literatura, principalmente, no que se refere ao trabalho em equipe. Sedelmaier e Landes (2014) afirmam que somente aqueles indivíduos capazes de colaborar são competentes para resolver os problemas enfrentados diariamente pelos engenheiros de software. Esta visão alinha-se ao mercado de trabalho, visto que o processo de desenvolvimento de software ocorre em equipes e muitas vezes estão distribuídas em diferentes países, onde trabalhar em equipe se torna fundamental.

"Resolução de problemas e situações difíceis", "Comunicação oral e escrita", "Adaptação a mudanças" e "Criatividade" também foram avaliadas com alto grau de importância. Estão alinhadas ao mercado de trabalho, pois atualmente são necessárias para a resolução dos problemas, para saber lidar com situações complexas não somente no âmbito técnico, mas também em relação às equipes. Estar preparado para as mudanças que ocorrem em um projeto, bem como nos requisitos de um sistema durante 
seu processo de desenvolvimento e estar apto a pensar de forma criativa para encontrar as melhores soluções dentro do cenário em que esta necessidade se apresente.

Tabela 1. Importância no desenvolvimento de carreira

\begin{tabular}{|l|l|c|c|c|c|c|c|}
\hline \multicolumn{2}{|c|}{ Soft Skills } & 1 & 2 & 3 & 4 & 5 & SCO \\
\hline S-01 & Trabalhar em equipe. & 0 & 1 & 0 & 9 & 31 & 0 \\
\hline S-02 & Comunicação oral e escrita. & 0 & 0 & 5 & 16 & 20 & 0 \\
\hline S-03 & Organização da sua rotina de trabalho. & 0 & 2 & 5 & 18 & 16 & 0 \\
\hline S-04 & Resolução de problemas e situações difíceis. & 0 & 0 & 9 & 11 & 21 & 0 \\
\hline S-05 & Adaptação a mudanças. & 0 & 0 & 7 & 13 & 20 & 1 \\
\hline S-06 & Pensamento Crítico. & 0 & 3 & 8 & 14 & 16 & 0 \\
\hline S-07 & Criatividade. & 0 & 2 & 10 & 11 & 17 & 1 \\
\hline S-08 & Liderança. & 0 & 2 & 9 & 19 & 11 & 0 \\
\hline S-09 & Responsabilidade. & 0 & 0 & 6 & 9 & 26 & 0 \\
\hline S-10 & Autodidata. & 1 & 4 & 13 & 11 & 11 & 1 \\
\hline S-11 & Moral ética e profissional. & 1 & 1 & 4 & 13 & 22 & 0 \\
\hline S-12 & Planejamento. & 0 & 1 & 7 & 17 & 16 & 0 \\
\hline S-13 & Negociação. & 0 & 4 & 11 & 14 & 12 & 0 \\
\hline S-14 & Habilidades interpessoais. & 0 & 5 & 6 & 15 & 15 & 0 \\
\hline S-15 & Resolução de conflitos. & 0 & 3 & 9 & 15 & 14 & 0 \\
\hline S-16 & Capacidade analítica. & 0 & 0 & 8 & 14 & 17 & 2 \\
\hline S-17 & Trabalhar de forma independente. & 1 & 5 & 15 & 9 & 10 & 1 \\
\hline S-18 & Visão do negócio & 0 & 2 & 12 & 13 & 14 & 0 \\
\hline S-19 & Foco no cliente & 0 & 1 & 4 & 17 & 18 & 1 \\
\hline S-20 & Empatia & 0 & 2 & 3 & 18 & 17 & 1 \\
\hline S-21 & Tomada de decisão & 0 & 1 & 7 & 14 & 18 & 1 \\
\hline
\end{tabular}

Como soft skill avaliada com menor grau de importância verificou-se "Trabalhar de forma independente". Isso pode ser ocasionado pela falta de entendimento sobre em que momento esta seria aplicada no cenário da engenharia de software. Também pela forma de trabalho das empresas, que talvez possuam processos rígidos, que fazem com que os profissionais não tenham a necessidade de utilizar-se desta soft skill.

Outra soft skill percebida com baixa importância pelos respondentes e de certa forma surpreendente é a soft skill "Autodidata". Considera-se este resultado como um ponto de atenção e preocupação, tendo em vista que as características da área conhecimento utilizam-se desta habilidade para aprender novos conhecimentos e se manter atualizado constantemente.

\subsection{Análise da Importância no Desenvolvimento de Atividades Diárias}

Quando se analisa a importância das soft skills para desenvolvimento de atividades diárias, percebe-se que "Trabalhar em equipe", "Responsabilidade" e "Moral ética e profissional" continuam em destaque. Porém, outras juntam-se aos achados da presente pesquisa como essenciais. São elas: "Comunicação Oral e escrita", "Adaptação a mudança", "Resolução de conflitos", "Capacidade analítica", e "Tomada de decisão". 
Tabela 2. Importância no desenvolvimento de atividades diárias

\begin{tabular}{|l|l|c|c|c|c|c|c|}
\hline \multicolumn{2}{|c|}{ Soft Skills } & 1 & 2 & 3 & 4 & 5 & SCO \\
\hline S-01 & Trabalhar em equipe. & 0 & 2 & 2 & 11 & 22 & 0 \\
\hline S-02 & Comunicação oral e escrita. & 1 & 0 & 5 & 12 & 19 & 0 \\
\hline S-03 & Organização da sua rotina de trabalho. & 0 & 3 & 5 & 19 & 10 & 0 \\
\hline S-04 & Resolução de problemas e situações difíceis. & 1 & 0 & 5 & 14 & 17 & 0 \\
\hline S-05 & Adaptação a mudanças. & 1 & 0 & 6 & 12 & 18 & 0 \\
\hline S-06 & Pensamento Crítico. & 0 & 5 & 8 & 11 & 12 & 1 \\
\hline S-07 & Criatividade. & 1 & 3 & 12 & 9 & 12 & 0 \\
\hline S-08 & Liderança. & 1 & 4 & 9 & 14 & 9 & 0 \\
\hline S-09 & Responsabilidade. & 0 & 1 & 5 & 11 & 20 & 0 \\
\hline S-10 & Autodidata. & 1 & 0 & 10 & 14 & 11 & 1 \\
\hline S-11 & Moral ética e profissional. & 0 & 2 & 7 & 9 & 17 & 1 \\
\hline S-12 & Planejamento. & 1 & 1 & 10 & 14 & 10 & 1 \\
\hline S-13 & Negociação. & 1 & 4 & 10 & 9 & 12 & 1 \\
\hline S-14 & Habilidades interpessoais. & 1 & 3 & 8 & 12 & 12 & 1 \\
\hline S-15 & Resolução de conflitos. & 1 & 4 & 3 & 12 & 17 & 0 \\
\hline S-16 & Capacidade analítica. & 0 & 4 & 5 & 9 & 19 & 0 \\
\hline S-17 & Trabalhar de forma independente. & 1 & 4 & 11 & 7 & 14 & 0 \\
\hline S-18 & Visão do negócio & 2 & 2 & 7 & 12 & 13 & 1 \\
\hline S-19 & Foco no cliente & 1 & 1 & 5 & 14 & 15 & 1 \\
\hline S-20 & Empatia & 2 & 1 & 7 & 10 & 15 & 2 \\
\hline S-21 & Tomada de decisão & 2 & 0 & 7 & 9 & 18 & 1 \\
\hline
\end{tabular}

\subsection{Análise da Frequência de Uso de Soft Skills}

Quanto à frequência percebe-se que enquanto a necessidade do uso de soft skills é diária (Figura 1), a preocupação dos profissionais com o desenvolvimento dessas habilidades acontece semestralmente ou anualmente (Figura 2).

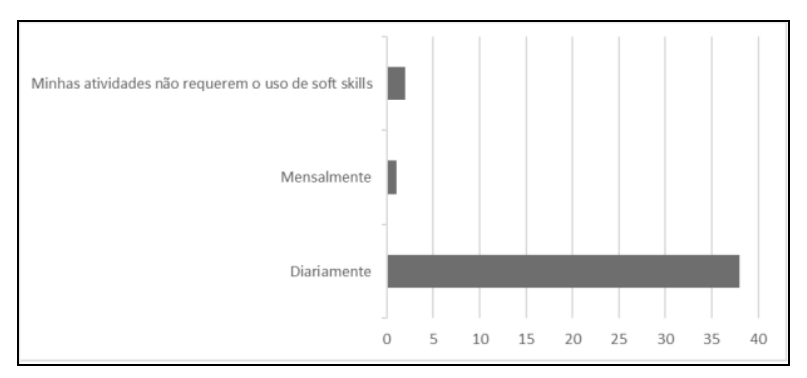

Figura 1. Frequência de uso de soft skills

Dessa forma pode levar a uma defasagem no desenvolvimento das habilidades necessárias em atividades diárias e na carreira, ocasionando demanda de tempo maior para se desenvolver-se na carreira e apresentar dificuldade na execução de suas atividades. Gera-se um prejuízo para os profissionais que podem acabar frustrados com suas carreiras não desenvolvendo-se na velocidade que gostariam e para as empresas, pois o tempo que as atividades necessitam para serem executadas podem aumentar em 
função de falta de soft skills desenvolvidas. Também pode gerar atrasos na preparação de novos membros da equipe, pois podem ser afetados pelo nível de soft skills de membros antigos, gerando uma cultura de não se preocupar em desenvolvimento com a devida importância ou frequência, em que as competências técnicas são desenvolvidas.

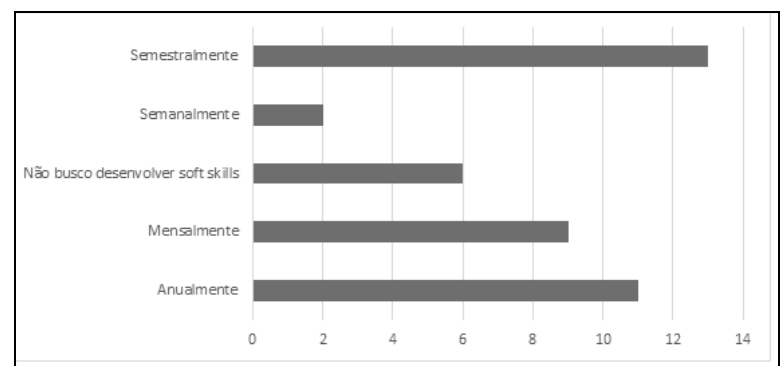

Figura 2. Frequência de desenvolvimento de soft skills

Entre profissionais que buscam treinamentos, 20 fazem uso de modalidades presenciais ou online. Ainda, 8 registraram que fazem o uso de vídeos na Internet para desenvolver este tipo de habilidade. Dentre as formas de incentivo apresentadas pelas empresas, a maioria segundo os respondentes (24), fornecem esses treinamentos, o que está alinhado com a forma que os profissionais procuram desenvolver-se neste quesito.

\section{Considerações Finais}

Essa pesquisa analisou a importância das soft skills sob o ponto de vista de profissionais da área de Engenharia de Software, por meio de uma survey cujo instrumento foi elaborado com base na literatura e validado por pré-testes, antes da coleta de dados. Os resultados se mostraram satisfatórios para agregar conhecimento do ponto de vista dos profissionais em relação ao desenvolvimento de soft skills, bem como quanto à importância que elas possuem em atividades diárias e na carreira nessa área.

Contribui para a área de Engenharia de Software, pois através do mapeamento dessas soft skills usadas com maior frequência e que possuem impacto na vida dos engenheiros de software pode-se incentivar e trabalhar o desenvolvimento delas, gerando profissionais mais preparados para produzir software de qualidade. Contribui com os profissionais da área, pois conhecendo as soft skills necessárias podem se preparar para desenvolver melhor suas atividades e suas carreiras, atingindo assim um nível maior de satisfação profissional.

Pode-se considerar que existem alto grau de importância de soft skills na visão dos profissionais da amostra. Porém notou-se que não existe o mesmo grau de preocupação quanto ao desenvolvimento destas habilidades pelos respondentes. Trabalhar em equipe, responsabilidade e moral ética e profissional mostraram-se as soft skills, com maior importância. Não houve uma diferença significativa em relação aos resultados, quando a análise foi feita somente com aqueles que possuem mais de 1 ano de experiência, o que demonstra que mesmo profissionais menos experientes possuem uma visão alinhada das habilidades, que contribuem para a carreira e atividades diárias.

Entre as limitações encontradas têm-se a quantidade de respostas obtidas e ainda a dificuldade de compreensão de cada soft skill por parte dos respondentes, pois podem ter tido diferentes entendimentos, visto que algumas são mais subjetivas. Como pesquisas futuras sugere-se a replicação dessa survey em outras amostras para expandir 
a validade externa ou generalizar os resultados. Uma análise aprofundada dessas soft skills, com diferentes equipes em início de carreira e experientes em desenvolvimento de software. Pesquisas sobre o impacto no desenvolvimento e na qualidade de software.

\section{Referências}

Ahmed, F., Capretz, L. F., Bouktif, S. and Campbell, P. (2013) "Soft Skills and Software Development: A Reflection from Software Industry", Electrical and Computer Engineering Publications, p. 40.

Calazans, A. T. S., Paldês, R. A., Braosi, E., Rezende, K. M. and Pereira, N. I. (2017) "O perfil do analista de requisitos de software: uma comparação entre a academia e o mercado de trabalho brasileiro", Centro Universitário de Brasília, Distrito Federal, Brasil.

Cooper, D. and Schindler, P. (2003) "Método de pesquisa em Administração", Bookman.

Dutra, J. S. (2010) "Competências: conceitos e instrumentos para a gestão de pessoas na empresa moderna", Atlas.

Fleury, M. T. L. and Fleury, A. (2001) "Construindo o conceito de competência", https://dx.doi.org/10.1590/S1415-65552001000500010.

Froehlich, M. B. (2016) “Análise das Soft Skills no Gerenciamento de Projetos de Service Desk" Trabalho de Conclusão de Curso (Graduação em Sistemas de Informação), Universidade do Vale do Rio dos Sinos, São Leopoldo, Rio Grande do Sul.

Gerhardt, T. E and Silveira, D. T. (2009) "Métodos de pesquisa", http://www.ufrgs.br/cursopgdr/downloadsSerie/derad005.pdf.

Gimenes, I. M. S. (2015) “Os Dilemas Didáticos da Engenharia de Software”, Revista da Sociedade Brasileira de Computação - Brasil, Ed. 28.

González-Morales, D., Antonio, L. M. M. and García, J. L. R. (2010) "Teaching 'Soft' Skills in Software Engineering”, Escuela Técnica Superior de Ingeniería Informática - ETSII Universidad de La Laguna, ULL La Laguna, Tenerife, Spain.

Peters, J. F. and Pedrycz, W. (2001) "Engenharia de Software: Teoria e Prática", Campus.

Rao, M. S. (2012) "Myths and truths about soft skills", Association for Talent Development, https://www.td.org/magazines/td-magazine/myths-and-truths-aboutsoft-skills.

Sedelmaier, Y. and Landes, D. (2014) "Software Engineering Body of Skills (SWEBOS)", Faculty of Electrical Engineering and Informatics, University of Applied Sciences and Arts, Coburg, Germany.

Sommerville, I. (2011) “Engenharia de Software”, Pearson Prentice Hall.

Wang, Y. and King, G. (2000) "Software Engineering Process Principles and Applications", CRC Press LLC. 